\author{
Summary Report \\ High-Energy Physics Outstanding Junior Inves- \\ tigator Program \\ PI: David E. Kaplan \\ 6/30/03 - 06/30/08 \\ Organization: Johns Hopkins University \\ 3400 N. Charles Street \\ Baltimore, MD 21218 \\ Award Number: DE-FG02-03ER41271 \\ Unexpended funds: $\$ 0$ (estimated)
}

\title{
1 Overview
}

Throughout the past five years I have worked to uncover what physics lies beyond that of the standard model. My main focus in the first two and a half years has been to understand physics at the electroweak scale, and to a lesser extent understand the relationship between particle physics and cosmology. My final two and a half years was spent on studying the feasibility of discovering "non-standard" models of electroweak physics at hadron colliders, working in close contact with experimentalists at the Tevatron and the LHC.

My biggest successes during this period has been both in electroweak physics - expanding our understanding of the Higgs sector in supersymmetric theories and ultraviolet completions of little Higgs theories - and in collider physics discovering a method for identifying high momentum top quarks and realizing the potential for LHCb to discover some versions of supersymmetry. I have also made some progress towards a particle physics/effective field theory solution of the cosmological constant problem.

\section{Collider Physics}

Over the past year, I have shifted my focus to expanding the discovery potential of hadron colliders. Our postdocs, students and I have discovered a new method of identifying high- $p_{T}$ top quarks in the hadronic channel above background ('top tagging'), which will dramatically aid in the search for new $t-\bar{t}$ resonances, a common feature of extra dimensional theories. My students and I have also studied the feasibility of discovering certain Higgs decays and supersymmetric particle production using $b$-physics triggers.

A failure of indications of new physics from indirect tests suggests that if new physics beyond a Higgs boson is involved with electroweak symmetry breaking, new states should arise with masses above a TeV. Heavy states coupled to top quarks would produce highly boosted jets, with the top decay products collimated. Such a final state is not picked up efficiently by standard search methods. Postdocs Matt Schwartz and Brock Tweedie, and graduate student 
Keith Rehermann, and I discovered an algorithm from which a hadronically decaying top quark at high $p_{T}$ can be distinguished from a QCD jet with an efficiency similar to that of bottom-jet tagging at the Tevatron at much lower $p_{T}$. The algorithm proved so effective on Monte Carlo samples, it has now been implemented as part of the CMS experiment's 'toolkit' used to search for new physics. The efficiency is even high enough that the LHC could now have sensitivity to resonances up to $2 \mathrm{TeV}$ with only a few inverse femtobarns of data.

As discussed below, more natural theories of electroweak symmetry breaking, and specifically of supersymmetry, involve Higgs bosons which decay in ways different from the standard model Higgs. In addition, supersymmetry with R-parity violation is much less constrained than the standard version and differs significantly phenomenologically from standard supersymmetry. These new signatures often involve new or multiple displaced vertices and may require different techniques for their discovery, as the standard searches (requiring jets with high transverse momentum and/or large transverse missing energy) will not be capable of picking out such signatures.

My student Keith Rehermann and I investigated the ability for the LHCb detector at CERN to discover supersymmetry if baryon number is violated [1]. In such theories, the lightest superpartner (which we take to be a neutralino) has a macroscopic decay length in nearly all of the allowed parameter space, and for a large portion it should decay within a typical detector. The LHCb is ideally suited for such studies as the detector is designed to pick out $b$-decays via a non-zero impact parameter. We have found that the LHCb, after some level of cuts, could see up to $10^{5}$ neutralinos from squark production in the first year. The LHCb could be sensitive to squark masses up to nearly $1 \mathrm{TeV}$. In addition, a Higgs that decays to (subsequently decaying) neutralinos would be seen in nearly all of the parameter space. The ATLAS and CMS detectors would have a much weaker sensitivity to these signals as they do not as of yet plan to have low-level displaced vertex triggers, and the lack of missing transverse energy and isolated leptons (because of the large multiplicity of jets) will drastically weaken the standard search strategies. Thus, if R-parity is violated, the LHCb may be the first to discover supersymmetry!

\section{Supersymmetric Theories}

During the past four years, I have debunked a long-standing belief about the Higgs mass in supersymmetric theories, constructed a new mechanism of supersymmetrybreaking which could be applied more generically, and have found a class of supersymmetric parameter space which not significantly constrained by current data and implies the existence of Higgs production hidden in the LEP data!

The lore about supersymmetric extensions of the standard model has been that it is impossible to generate a mass for the Higgs near or above $200 \mathrm{GeV}$ with restrictions of "naturalness" and perturbativity up to the Planck scale placed on all couplings. My collaborators (Puneet Batra, Antonio Delgado and Tim Tait) and I have discovered a class of supersymmetric theories which are perturbative 
up to the GUT scale and yet allow for Higgs masses well above $300 \mathrm{GeV}$. The key ingredient is the coupling of the Higgs to an asymptotically free gauge group. The results are published in an article [4]. In a second paper [5], we explored the non-standard Higgs phenomenology of some of these models and found bizarre regions of parameter space which were allowed, e.g., $\tan \beta<1$. The Higgs phenomenology of these models is distinct from those supersymmetric models already thoroughly explored and has different implications for the LHC Higgs searches. No longer does the discovery of a heavy Higgs rule out supersymmetry and weakly coupled unification, but it automatically implies the existence of new gauge bosons in the few $\mathrm{TeV}$ mass range.

With collaborators Patrick Fox and (my student) Linda Carpenter, I have found a way of communicating supersymmetry breaking to the minimal supersymmetric standard model which solves the flavor, CP, mu and Higgs mass problems. It is the MSSM with a singlet which couples to the hidden sector through both anomaly mediation and with a direct coupling to a hidden sector $U(1)$. The direct coupling gives a dirac mass to the hypercharge gaugino, yet only induces scalar masses via finite loops. The result is an ultra-violet insensitive superpartner spectrum so it is not adversely affected by new high-scale flavor physics. It also automatically corrects the slepton mass (tachyon) problem in anomaly mediation by generating a positive squared mass for all scalars which carry hypercharge. An extra quartic is generated for the Higgs potential through coupling to the singlet thus easily pushing the Higgs mass above the current LEP bound. The model predicts significant CP-violation near the experimental limits and measurements there are correlated to the spectrum discovered at the LHC. However, the minimal model turns out to be fine-tuned to the extent that other realistic models with supersymmetry mediated by gravity are, and, in addition, unstable due to the gauge and global singlet required. It is possible to ameliorate these problems by adding a second field and divide the task of successful electroweak symmetry breaking and a Dirac Bino mass. The results are in a preprint [6] and have been submitted for publication.

With Hopkins students Carpenter and E.J. Rhee, I investigated supersymmetric theories with R-parity and baryon number violation [7]. These theories have enormous regions in parameter space in which the dominant decay of the lightest Higgs boson is into six jets via two on shell neutralinos. Such a Higgs does not show up in any exclusive search at LEP and its mass is constrained by the model-independent Higgs search done only by the OPAL collaboration, which sets a weak bound of $82 \mathrm{GeV}$. This essentially unexplored region of parameter space allows for much more natural theories of softly broken supersymmetry with a Higgs mass about equal to the $Z$ boson mass. Such a Higgs could be extremely difficult to discover at the LHC and thus new analyses of LEP data are warranted.

The fine-tuning found generically in models of supersymmetry breaking which solve flavor problems have lead us (myself and some students) to survey a more general parameter space of a supersymmetric standard model to ask whether the general scenario itself is disfavored. The result will either be the realization that the electroweak symmetry breaking cannot be reasonably 
explained in the minimal model, or the discovery of new unexplored parameter space phenomenologically which is currently favored by experiment.

\section{Little Higgs Theories}

Little Higgs theories [8] are charged with solving the "little hierarchy problem", i.e., to explain the lack of indirect evidence of new physics at the weak scale without forcibly moving that scale up by an order of magnitude and thereby requiring fine tuning. Such theories break down not too much farther above the weak scale (typically below $10 \mathrm{TeV}$ ), and thus these models would be rendered more compelling if clear candidates for ultraviolet completions existed. Unfortunately, technicolor-like completions are difficult at best and require a number of dynamical assumptions. New ideas in this direction would change the face of these theories. The best possibility for a strongly coupled example would be suggested by Randall-Sundrum-like scenarios [9], and thus exploration of marrying these two ideas have been underway recently [10]. I, on the other hand, have explored a more ambitious approach, namely a natural weakly coupled completion.

Along with Puneet Batra, a former student of mine and currently a postdoc at Columbia, I have successfully constructed a two-stage little Higgs "tower". The implication is a model which is weakly coupled, non-supersymmetric and has a cutoff of around $100 \mathrm{TeV}$ with a naturally light (order $100 \mathrm{GeV}$ ) Higgs mass. This is accomplished my embedding a little Higgs model into a larger version of the same type (specifically, the SU(4) model into an SU(12) model). Unfortunately, while all of the parametrically large contributions to the Higgs mass are cancelled, the normally considered "safe" loops turn out to be numerically large. We argue that generically it is difficult to perturbatively complete the little Higgs beyond $10 \mathrm{TeV}$ without fine tuning due to numerical factors in the standard model (dominantly the top Yukawa and number of colors). At the same time, we outline a structure which produces a toy model of a so-called "tumbling gauge theory" in which naturally light scalars exist in a weakly coupled theory with a cutoff five orders of magnitude or so above their masses. These models still have scales put in by hand and thus we are looking for a fully dynamically mechanism. However, the ability to cancel all dangerous contributions to scalar masses has greatly encouraged us. The work has been published in [11]. A precursor to this work (a "one-and-a-half" stage little Higgs tower) by M. Schmaltz, W. Skiba, and myself has been published in [12].

\section{$5 \quad$ Dark Energy}

Raman Sundrum and I have recently proposed a partial solution to the cosmological constant problem [14]. The most significant part of the problem has always been that matter loops renormalize the cosmological constant and there doesn't seem to be a symmetry protecting it below the TeV scale which is may 
orders of magnitude above the apparent value. The possibility of new physics in the gravitational sector at short distances [13] fails to deal with the fact that loop diagrams of matter involve only the couplings of long wavelength gravitational fields to the quantized standard model, a domain in which general relativity appears to work perfectly well. What we have done is to combine both a symmetry of matter and a low cutoff on gravity to allow for a tiny, but technically natural, cosmological constant. The symmetry is called "energy-parity" and requires the introduction of a partner standard model with a wrong-sign Lagrangian (including kinetic terms). The symmetry is softly broken by gravity, which becomes the source of an instability in the "ghost" sector. The cutoff in the gravity sector acts to regulate this instability, as well as quantum gravity corrections to the cosmological constant. In this paradigm, the size of the cosmological constant is relegated to as of yet untested short distance physics and thus is completely natural in the effective theory. Amazingly enough, phase transitions are trivial to understand in such a theory and models of inflation work perfectly well to generate the initial conditions of standard Big Bang cosmology with an empty ghost sector. The result is a number of possible experimental signals which we are currently exploring, such as modifications of gravitational interactions at the sub-millimeter scale, medium to very high energy cosmic ray photons, and gravitational Lorentz violation. We are also exploring possible effects on cosmological evolution. In addition, we are examining toy models of UV completions to both the gravitational sector and theories with ghost degrees of freedom.

\section{References}

[1] D. E. Kaplan and K. Rehermann, arXiv:0705.3426 [hep-ph].

[2] For an overview of the most current precision tests of the standard model (including Tevatron data), see the LEP Electroweak Working Group web page at http://lepewwg.web.cern.ch/LEPEWWG/

[3] See for example recent talks by C. Ciobanu and R. Hauser at Moriond, http://moriond.in2p3.fr/EW/2006/Transparencies/index.html

[4] P. Batra, A. Delgado, D. E. Kaplan and T. M. P. Tait, JHEP 0402, 043 (2004) [arXiv:hep-ph/0309149].

[5] P. Batra, A. Delgado, D. E. Kaplan and T. M. P. Tait, JHEP 0406, 032 (2004) [arXiv:hep-ph/0404251].

[6] L. Carpenter, P. J. Fox and D. E. Kaplan, arXiv:hep-ph/0503093.

[7] L. M. Carpenter, D. E. Kaplan and E. J. Rhee, arXiv:hep-ph/0607204, Phys. Rev. Lett., to appear.

[8] For a review, see M. Schmaltz and D. Tucker-Smith, arXiv:hep-ph/0502182. 
[9] L. Randall and R. Sundrum, Phys. Rev. Lett. 83, 3370 (1999) [arXiv:hep$\mathrm{ph} / 9905221]$.

[10] K. Agashe, R. Contino and A. Pomarol, Nucl. Phys. B 719, 165 (2005) [arXiv:hep-ph/0412089].

[11] P. Batra and D. E. Kaplan, JHEP 0503, 028 (2005) [arXiv:hep$\mathrm{ph} / 0412267]$.

[12] D. E. Kaplan, M. Schmaltz and W. Skiba, Phys. Rev. D 70, 075009 (2004) [arXiv:hep-ph/0405257].

[13] R. Sundrum, Phys. Rev. D 69, 044014 (2004) [arXiv:hep-th/0306106].

[14] D. E. Kaplan and R. Sundrum, JHEP 0607, 042 (2006) [arXiv:hepth/0505265]. 